\title{
The Association between Childhood Traumatic Experiences and Suicidality in College Students
}

\author{
Hyun JuYang, MD, PhD, Young Eun Jung, MD, PhD, Joon Hyuk Park, MD, PhD, \\ Moon Doo Kim, MD, PhD \\ Department of Psychiatry, Jeju National University Hospital, Jeju National University School of Medicine, Jeju, Korea
}

\begin{abstract}
Background: To address the high suicide rate in Korea, determining the suicidality risk factors is a key concern in policymaking and prevention. This study aimed to describe the effects of traumatic childhood experiences on suicidality among young adults.

Methods: This study included 1,379 subjects from two universities in Jeju, Korea. All participants completed selfadministered questionnaires that included demographic variables, the Adverse Childhood Experiences (ACE) scale to evaluate traumatic childhood experiences, and a Korean version of the Suicide Module of the Mini International Neuropsychiatric Interview to evaluate suicidality.

Results: Of the subjects, 33.2\% reported experiencing traumatic childhood experiences, and the prevalence of positive suicidality was $21.8 \%$. Regression analyses indicated a significant association between traumatic childhood experiences and positive suicidality after controlling for multiple variables. Furthermore, subjects with positive suicidality were likely to report experiencing more traumatic childhood experiences $(p<0.001)$. After categorizing the subjects into two groups according to ACE score, the positive ACE group showed a higher risk of suicidal ideation $(p<0.001$, adjusted odds ratio $[A O R]=2.09)$ and suicidal attempt $(p<0.001, A O R=4.15)$ than those negative ACE.

Conclusion: Intervention and practical strategies for college students with a history of traumatic childhood experiences must be considered for suicide prevention.
\end{abstract}

Keywords Traumatic childhood experiences; College students; Suicide

\section{INTRODUCTION}

Given that more than one million people worldwide commit suicide annually, countries prioritize suicide prevention as the most crucial public health project. The suicide rate in South Korea is particularly high and has increased gradually. According to a summary of a 2020 suicide prevention white book, in 2018, the total number of suicide cases and the average daily suicide rate were 13,670 and $37.5 \%$, respectively. The suicide rate per 100,000 people was 26.6 , ranking fifth among the most common causes of death. These data are the highest among the OECD countries. Data of Statistics Korea
(KOSTAT) revealed in 2019 that suicide was the leading cause of death for young adults or those in their teens to thirties [1]. The high suicide rate of young adults is a prominent social problem, yet little is known about how the risk for young adult suicide develops during earlier life stages. Once suicide is committed successfully, it cannot be undone, thus predictors of suicide attempts among the young adults should be analyzed comprehensively to identify high-risk groups and determine interventions $[2,3]$.

Traumatic childhood experience is a strong predictor of suicide attempts for those who undergo suicide attempts or suicidal ideation [4]. It disturbs healthy de-

Received July 6, 2021, Revised August 24, 2021, Accepted August 24, 2021

Correspondence: Moon Doo Kim, MD, PhD

Department of Psychiatry, Jeju National University School of Medicine, 15 Aran 13-gil, Jeju 63241, Korea

TEL +82-64-717-1850 FAX +82-64-717-1849 E-mail mdkim66@jejunu.ac.kr ORCID https://orcid.org/0000-0002-6441-630X

Copyright $@$ by Korean Society for Affective Disorders.

This is an Open Access article distributed under the terms of the Creative Commons Attribution Non-Commercial License (http://creativecommons.org/licenses/ by-nc/4.0/) which permits unrestricted non-commercial use, distribution, and reproduction in any medium, provided the original work is properly cited. 
velopment, increases suicide risk in adolescence, causes mental illnesses (e.g., depression, alcohol abuse, drug addiction, and suicidal behaviors), and has significant effects on suicide attempts. A study by the Foundation for Preventing Youth Violence of teenagers in South Korea reported that $42.9 \%$ of teenagers who had childhood traumatic experiences showed suicidal ideation and that $41.8 \%$ of them attempted to commit suicide [5]. Such ratios were higher than those in a group of teenagers who did not have childhood traumatic experiences. Furthermore, it was found that traumatic childhood experiences consistently had negative effects on people beyond adolescence and into adulthood. Other than existing studies conducted based on clinical and high-risk groups, general, cross-sectional, and longitudinal research based on ordinary people in local communities also verified that traumatic childhood experiences were closely related to suicidal behaviors [6]. A study conducted among adults in South Korea indicated that $62.2 \%$ of respondents with traumatic childhood experiences thought about committing suicide and that $28.3 \%$ of them had made suicide attempts [7]. These rates are significantly higher than the suicide rate of $0.03 \%$ in South Korea in 2018, as announced by the KOSTAT.

It was also reported that those who encountered interpersonal traumatic events suffered from more severe trauma, performed more evasive actions, and experienced more negative effects than those who encountered non-interpersonal traumatic events (e.g., natural disasters and accidental events) [8]. One main characteristic of interpersonal traumatic events in childhood is that these events include physical, sexual, or emotional abuse performed by those with parental authority over or close acquaintanceship with them when they are in a developmentally vulnerable or critical state. Consequently, these events significantly affect the attachment formation, development, and maturity of an individual. Interpersonal trauma occurs in a repeated, chronic, and prolonged manner and shows complex properties over time [9]. The scale of damage caused by interpersonal trauma is wider than that of simple trauma and negatively affects individuals, ranging from children to adults, thus it can be related to a risk of occurrence of various mental illnesses [10], such as posttraumatic stress disorder, depressive disorders, anxiety disorders, and risk of occurrence of suicidal behaviors [11-13].

However, arguments can be raised while analyzing the results of the aforementioned studies, in that subjects of all ages were targeted in these studies and that the definition of traumatic experience varied. Therefore, the current study classified traumatic childhood experiences and analyzed a relationship between traumatic childhood experiences and suicidality of college students who represent the young adult group. Based on the classification and analysis, it developed a broad-range mediation method for preventing college students from committing suicide and performing early interventions before suicidal attempts.

\section{MATERIALS AND METHODS}

\section{Subjects}

We included college students aged 18 years or older from two colleges in the Jeju, Korea as eligible subjects. Initially, 1,397 subjects were recruited through contact with the health promotion centers at each university. From these, $98.7 \%(n=1,379)$ of respondents who completed the questionnaire were included in the final analysis. All subjects were fully informed about the study protocol and written statements of informed consent were signed by the subjects. The study was approved by the Institutional Review Board of Jeju National University Hospital (IRB no. JNUH 2018-10-009).

\section{Assessment}

The survey consisted of self-administered questionnaires. Questions involved various sociodemographic variables (age, sex, parental marital status, self-assessed living standard, academic achievement, satisfaction with familial relationship, previous neuropsychiatric history, and previous bullying history) and a self-reported assessment. Trained assistants explained the purpose of the survey to the participants and provided directions for participants to answer the survey questionnaires, which included several self-reported screening instruments.

To obtain information regarding traumatic childhood experiences, we administered the Adverse Childhood Experiences (ACE) scale (Supplementary Table). The ACE scale is composed of 10 items - including abuse (childhood emotional, physical, and sexual abuse), neglect (childhood emotional and physical neglect), and household dysfunction (parental separation or divorce, mother treated violently, household substance abuse, 
household mental illness, and incarcerated household member) - and was used as a measurement of exposure to traumatic stress during childhood. Exposure to any ACE category counts as one point, and the score is a summation of exposure to each adverse experience during the respondents' first 18 years of life [11].

This study employed the Korean version of the suicide module of the Mini International Neuropsychiatric Interview (M.I.N.I.) to screen for suicidality. M.I.N.I. is a structured interview schedule that screens for various psychiatric disorders and the validity and reliability of the Korean version of this measure have neem confirmed. The suicide module of the M.I.N.I. includes six questions assessing suicidal ideation within the past month ("Have you thought you would be better off dead or wished you were dead;" "Have you wanted to harm yourself;" and "Have you thought about suicide?"), suicide plans within the past month ("Did you have a suicide plan?"), suicide attempts within the past month ("Have you attempted suicide?"), and lifetime suicide attempts ("In your lifetime, have you ever attempted suicide?"). The scores for each answer were weighted according to its importance in assessing suicide risk, and the total score was used to classify current suicide risk as low (1-5 points), moderate (6-9 points), or high ( $>9$ points). In the present study, we defined 1 point or higher as positive suicidality.

We additionally administered the Korean version of the Patient Health Questionnaire-9 (K-PHQ-9) and General Anxiety Disorder-7 (GAD-7) scale to evaluate mood and anxiety symptoms. The PHQ-9 is the nine-item depression component of the patient health questionnaire. It is a validated tool in mental health and can greatly assist clinicians when diagnosing depression [14]. The items of the PHQ-9 are based directly on the nine diagnostic criteria for major depressive disorder in the Diagnostic and Statistical Manual of Menta Disorders-fourth edition, text-revision (DSM-IV-TR). The total PHQ-9 score can range from 0 to 27 since each of the items can be scored from 0 ("not at all") to 3 ("nearly every day"). This study used the K-PHQ-9, as standardized by Han et al. [15], and defined five points as the cut-off for depressive symptoms. The K-PHQ-9 has been reported to have excellent internal consistency (Cronbach's $\alpha=0.88$ ) and validity with other depressive symptoms assessment tools [15]. Meanwhile, the GAD-7 score is calculated by evaluating responses of $0,1,2$, and 3 - "not at all," "several days," "more than half the days," and "nearly every day," respectively — and summating the scores for the seven questions. Scores of 5, 10, and 15 were taken as the cut-off points for mild, moderate, and severe anxiety, respectively. When used as a screening tool, further evaluation is recommended when the score is 10 or greater.

\section{Statistical analysis}

This study classified groups according to positive of suicidality and compared differences in sociodemographic variables and traumatic childhood experiences between the groups. The independent samples t-test and chi-squared test were performed for continuous variables and categorical variables, respectively. Descriptive statistics of continuous variables included means and standard deviation, and those of categorical variables included frequencies and ratios. Moreover, a multiple regression analysis was conducted to inspect the effects of types of traumatic childhood experiences on suicidality and sub-items of the M.I.N.I. SPSS 21.0 (IBM Corp., Armonk, NY, USA) was used to analyze data in this study. Results with $\mathrm{p}<0.05$ were considered significant.

\section{RESULTS}

Of the 1,379 subjects included in the analysis, $40.6 \%$ $(n=560)$ were male, and $59.4 \%(n=819)$ were female. The mean age of the included participants was $20.9 \pm 2.5$ years. In total, $78.2 \%(n=1,078)$ met the criteria for the "negative suicidality" group and $21.8 \%(n=301)$ for the "positive suicidality" group. The sociodemographic and emotion-related variables that differed significantly between the groups were sex $(p<0.001)$, parental marital status ( $p<0.001)$, self-assessed living standard $(p<0.001)$, and satisfaction with familial relationship $(\mathrm{p}<0.001)$. The trauma-related and clinical symptom-related variables that showed significant group differences were neuropsychiatric $(\mathrm{p}<0.001)$ and bullying $(\mathrm{p}<0.001)$ histories. In total scores for PHQ-9 $(p<0.001)$ and GAD$7(\mathrm{p}<0.001)$ also showed significant group differences (Table 1).

To identify any association between the subtype of ACE and suicidality, we performed multivariate logistic analysis, which was adjusted for age, sex, previous neuropsychiatric history, previous bullying history, the sum of the GAD-7, and the sum of the PHQ-9. After 
Table 1. Characteristics of the subjects

\begin{tabular}{|c|c|c|c|c|}
\hline \multirow{2}{*}{ Variable } & \multirow{2}{*}{ Total $(n=1,379)$} & \multicolumn{2}{|c|}{ Suicidality } & \multirow{2}{*}{$p$-value } \\
\hline & & No $(n=1,078)$ & Yes $(n=301)$ & \\
\hline Age, y & $20.9 \pm 2.5$ & $20.9 \pm 2.4$ & $20.9 \pm 2.8$ & 0.952 \\
\hline Sex & & & & $<0.001$ \\
\hline Male & $560(40.6)$ & $485(45.0)$ & $75(24.9)$ & \\
\hline Female & $819(59.4)$ & $593(55.0)$ & $226(75.1)$ & \\
\hline Parental marital status & & & & $<0.001$ \\
\hline Married & $1,075(78.0)$ & $866(80.3)$ & $209(69.4)$ & \\
\hline Divorced/separated/widowed & $304(22.0)$ & $212(19.7)$ & $92(30.6)$ & \\
\hline Self-assessed living standard & & & & $<0.001$ \\
\hline High & $268(19.4)$ & $223(20.7)$ & $45(15.0)$ & \\
\hline Middle & $949(68.8)$ & $757(70.2)$ & $192(63.8)$ & \\
\hline Low & $162(11.7)$ & $98(9.1)$ & $64(21.3)$ & \\
\hline Academic achievement & & & & 0.066 \\
\hline Above average & $407(29.5)$ & $313(29.0)$ & $94(31.2)$ & \\
\hline Average & $738(53.5)$ & $593(55.0)$ & $145(48.2)$ & \\
\hline Below average & $234(17.0)$ & $172(16.0)$ & $62(20.6)$ & \\
\hline Satisfaction with familial relationship & & & & $<0.001$ \\
\hline Good & $949(68.8)$ & $795(73.7)$ & $154(51.2)$ & \\
\hline Fair & $347(25.2)$ & $245(22.7)$ & $102(33.9)$ & \\
\hline poor & $83(6.0)$ & $38(3.5)$ & $45(15.0)$ & \\
\hline Previous neuropsychiatric history & $66(4.8)$ & $26(2.4)$ & $40(13.3)$ & $<0.001$ \\
\hline Previous bullying history & $191(13.9)$ & $95(8.8)$ & $96(31.9)$ & $<0.001$ \\
\hline \multicolumn{5}{|l|}{ Depressive symptoms } \\
\hline PHQ-9 total score & $4.1 \pm 4.4$ & $3.0 \pm 3.3$ & $8.0 \pm 5.5$ & $<0.001$ \\
\hline PHQ-9 positivity & $155(11.2)$ & $51(4.7)$ & $104(34.6)$ & $<0.001$ \\
\hline \multicolumn{5}{|l|}{ Anxiety symptoms } \\
\hline GAD-7 total score & $2.9 \pm 3.6$ & $2.0 \pm 2.7$ & $5.8 \pm 4.8$ & $<0.001$ \\
\hline GAD-7 positivity & $81(5.9)$ & $20(1.9)$ & $61(20.3)$ & $<0.001$ \\
\hline
\end{tabular}

Values are presented as number (\%) or mean \pm standard deviation for all variables. Different subtotal because of missing. PHQ-9, Patient Health Questionnaire-9; GAD-7, General Anxiety Disorder-7.

andependent t-test or chi-square test, as appropriate.

the analysis, any ACE was significantly associated with positive suicidality except physical neglect $(\mathrm{p}=0.124)$. Furthermore, an increased ACE score was associated with positive suicidality $(\mathrm{p}<0.001)$. Compared to the negative suicidality group, the positive suicidality group showed higher prevalence of ACE with three or more scores (Table 2).

To compare suicidal risk, the M.I.N.I. psychiatry suicidal risk items were defined by the ACE; participants were categorized into two groups. The "negative ACE group" comprised those whose scores indicating no ACE, while the "positive ACE group" comprised those who had at least one subtype of ACE. After multivariate logistic regression analysis, which was adjusted for age, sex, previous neuropsychiatric history, previous bullying history, parental marital status, self-assessed living standard, satisfaction with familial relationship, sum of the GAD-7, and sum of the PHQ-9, the prevalence rate for suicidal ideation within the past month was significantly higher in the positive than the negative ACE group ( $35.7 \%$ vs. $12.0 \%$; odds ratio [OR], $2.09 ; 95 \%$ confidence interval [CI], 1.45-3.02; $\mathrm{p}<0.001$ ), and the positive ACE group had higher rates of lifetime suicidal attempts than the negative ACE group (10.8\% vs $1.7 \%$; OR, $4.15 ; 95 \%$ CI, 2.03-8.50; $\mathrm{p}<0.001)$. There were significant group differences between members of the positive and negative ACE groups regarding the rate of those with above moderate suicidal risk ( $2.8 \%$ vs. $9.4 \%$; OR, $3.31 ; 95 \%$ 
Table 2. Results of multivariate logistic regression analysis of suicidality defined by M.I.N.I. suicidal risk items according to the types of ACE

\begin{tabular}{|c|c|c|c|c|c|}
\hline \multirow{2}{*}{ Prevalence of categories of ACE } & \multirow{2}{*}{$\begin{array}{c}\text { Total } \\
(n=1,379)\end{array}$} & \multicolumn{2}{|c|}{ Suicidality } & \multirow{2}{*}{$p$-value } & \multirow{2}{*}{$\begin{array}{l}\text { Adjusted } \mathrm{OR}^{\mathrm{a}} \\
\qquad(95 \% \mathrm{Cl})\end{array}$} \\
\hline & & No $(n=1,078)$ & Yes $(n=301)$ & & \\
\hline Emotional abuse & $185(13.4)$ & $93(8.6)$ & $92(30.6)$ & $<0.001$ & $2.08(1.40-3.09)$ \\
\hline Physical abuse & $163(11.8)$ & $77(7.1)$ & $86(28.6)$ & $<0.001$ & $2.94(1.93-4.46)$ \\
\hline Sexual abuse & $24(1.7)$ & $4(0.4)$ & $20(6.6)$ & 0.001 & $8.18(2.45-27.35)$ \\
\hline Emotional neglect & $103(7.5)$ & $31(2.9)$ & $72(23.9)$ & $<0.001$ & $4.25(2.49-7.25)$ \\
\hline Physical neglect & $14(1.0)$ & $3(0.3)$ & $11(3.6)$ & 0.124 & $3.42(0.71-16.37)$ \\
\hline Parent separation or divorce & $263(19.1)$ & $168(15.6)$ & $95(31.6)$ & 0.001 & $1.81(1.26-2.59)$ \\
\hline Mother treated violently & $37(2.7)$ & $15(1.4)$ & $22(7.3)$ & 0.031 & $2.54(1.09-5.92)$ \\
\hline Household substance abuse & $57(4.1)$ & $20(1.9)$ & $37(12.3)$ & $<0.001$ & $3.79(1.93-7.43)$ \\
\hline Household mental illness & $46(3.3)$ & $20(1.9)$ & $26(8.6)$ & 0.031 & $2.20(1.08-4.52)$ \\
\hline Incarcerated household member & $16(1.2)$ & $5(0.5)$ & $11(3.7)$ & 0.014 & $5.58(1.42-21.94)$ \\
\hline ACE score & & & & $<0.001$ & \\
\hline 0 & $920(66.8)$ & $802(74.5)$ & $118(39.3)$ & & \\
\hline 1 & 247 (17.9) & $183(17.0)$ & $64(21.3)$ & & \\
\hline 2 & $90(6.5)$ & $53(4.9)$ & $37(12.3)$ & & \\
\hline 3 & $52(3.8)$ & $18(1.7)$ & $34(11.3)$ & & \\
\hline$\geq 4$ & $68(4.9)$ & $21(1.9)$ & $47(15.7)$ & & \\
\hline
\end{tabular}

Values are presented as number (\%).

M.I.N.I., Mini International Neuropsychiatric Interview; ACE, Adverse Childhood Experiences; OR, odds ratio; $\mathrm{Cl}$, confidence interval; GAD-7, General Anxiety Disorder-7; PHQ-9, Patient Health Questionnaire-9.

${ }^{a}$ Adjusted for age, sex, previous neuropsychiatric history, previous bullying history, sum of GAD-7, and sum of PHQ-9.

Table 3. Comparisons of M.I.N.I. suicidal risk items between the group of positive and negative ACE

\begin{tabular}{|c|c|c|c|c|}
\hline \multirow{2}{*}{ Variable } & \multicolumn{2}{|c|}{ ACE } & \multirow{2}{*}{$p$-value } & \multirow{2}{*}{$\begin{array}{l}\text { Adjusted OR } \\
(95 \% \mathrm{Cl})\end{array}$} \\
\hline & Negative $(n=920)$ & Positive $(n=457)$ & & \\
\hline \multicolumn{5}{|l|}{ Past one month } \\
\hline Suicidal ideation & $110(12.0)$ & $163(35.7)$ & $<0.001$ & $2.09(1.45-3.02)$ \\
\hline Suicidal plan & $10(1.1)$ & $12(2.6)$ & 0.888 & $0.92(0.30-2.80)$ \\
\hline Suicidal attempt & $4(0.4)$ & $8(1.8)$ & 0.239 & $2.42(0.56-10.47)$ \\
\hline \multicolumn{5}{|l|}{ Lifetime } \\
\hline Suicidal attempt & $16(1.7)$ & $49(10.8)$ & $<0.001$ & $4.15(2.03-8.50)$ \\
\hline Above moderate suicidal risk & $26(2.8)$ & $43(9.4)$ & $<0.001$ & $3.31(1.80-6.11)$ \\
\hline
\end{tabular}

Values are presented as number (\%).

M.I.N.I., Mini International Neuropsychiatric Interview; ACE, Adverse Childhood Experiences; OR, odds ratio; $\mathrm{Cl}$, confidence interval; GAD-7, General Anxiety Disorder-7; PHQ-9, Patient Health Questionnaire-9.

${ }^{a}$ Adjusted for age, sex, previous neuropsychiatric history, previous bullying history, parental marital status, self-assessed living standard, satisfaction with familial relationship, sum of GAD-7, and sum of PHQ-9.

CI, 1.80-6.11; $\mathrm{p}<0.001)$ (Table 3).

\section{DISCUSSION}

This study was conducted to examine the relationship between traumatic childhood experiences and suicidality among college students who represent the young adult group. The analyses indicated that 21.8\%, 19.8\%, and $0.9 \%$ of participants belonged to the positive suicidality group, experienced suicidal ideation over the last month, and made suicide attempts over the last month, 
respectively. Meanwhile, 5.6\% of participants reported that they had attempted to commit suicide at least once. Despite difficulty directly comparing data due to the application of different test tools and subjects, these results were compared with results from other studies [1619]. It was found that the ratios calculated in this study were higher than the $20162.9 \%$ occurrence of suicidal ideation derived in a previous study, which was based on the evaluation of 5,102 members of the general population using the Korean version of the Composite International Diagnostic Interview. The values in the current study were also higher than the lifetime prevalence rate of suicidal ideation (9.2\%) and suicide attempts (2.7\%), as reported in a study by the World Health Organization [20], which was based on the results of 84,850 people from 17 countries who completed the Composite International Diagnostic Interview. The impact of women's traumatic childhood experiences on suicide risk increased when they encountered divorce, separation, or bereavement of their parents; when they suffered from low living conditions; when they had an unsatisfactory family relationship; when they had a history of psychiatric illnesses; or when they were bullied in the past. This was consistent with the results of existing studies [20-22].

In this study, $33.2 \%$ of participants had at least one type of traumatic childhood experience. Parental separation or divorce was the most reported traumatic childhood experience, followed by emotional abuse, physical abuse, and emotional neglect; traumatic childhood experiences were frequently in the form of hostile and rejective treatment (e.g., insults and threats for triggering feelings of contempt, insult, and humiliation) and direct and indirect abuse (e.g., beating caused by family troubles or trouble between the parents, verbal abuse mentally damaging children, and neglect of children). Traumatic childhood experiences, except for physical neglect, had an independently significant relationship with the suicidality of college and graduate students. Accordingly, a relationship between physical neglect and suicidality of college and graduate students should be re-analyzed with a sufficiently large sample.

Given that the impact of traumatic childhood experiences cannot be instantly recognized, people surrounding victims of traumatic childhood experiences are likely to overlook the severity of these experiences [10]. Issues related to these experiences become repeated and prolonged, thereby increasing their severity. Children cannot actively react to traumatic situations. As such, they tend to feel frustrated when they fail to find solutions for overcoming these situations. They are also likely to feel helpless, believing that they are incapable of managing these situations. When these traumatic experiences are repeated and prolonged, they have negative effects on the lives of sufferers that are then felt from childhood and even long-term into young adult [7]. Consequently, people with these experiences are likely to encounter problems that can be connected to suicidal urges, such as low self-esteem, insufficient problem-solving capacity, depression, high levels of aggression, personality changes, and a lack of social abilities [11,23]. Existing research limitedly focuses on physical or sexual abuse to analyze the effects of emotionally traumatic experiences in childhood on psychopathology in adulthood. Nevertheless, certain existing studies reported that emotional abuse experiences in childhood had a particularly high correlation with psychological maladjustment in adulthood among various abuse in childhood. These results imply that various traumatic experiences, including physical, sexual, and emotional abuse, are closely related to psychological maladjustment in young adult. In this study, the suicidality group reported that they frequently underwent traumatic childhood experiences, including physical abuse, emotional abuse, emotional neglect, and parental separation or divorce. This result is consistent with the aforementioned studies. Moreover, other studies stated that traumatic childhood experiences increased the lifetime suicide attempt rate in adulthood by approximately 1.5 times and that this rate increased approximately 30 times when these experiences were repeated 7 times or more. These results support our findings [11-13].

This study analyzed suicidal behavior by classifying them into suicidal ideation and attempts based on the M.I.N.I. In the analysis, the group of subjects without traumatic childhood experiences was compared with those who had traumatic childhood experiences. The analysis indicated that the group with traumatic childhood experiences showed a risk of suicidal ideation over the last month and that this group also showed a high lifetime suicide attempt rate and a higher suicide risk than the mean level. Suicidal ideation is regarded as a core risk factor for suicide attempts. A national comorbidity survey conducted in the United States of America reported that the possibility of suicidal ideation being transformed into suicide planning was $34 \%$ and of suicidal planning being transformed into suicide attempts was $72 \%$. Additionally, approximately $90 \%$ of 
unplanned suicide attempts and approximately $60 \%$ of planned suicide attempts among first suicide attempts occurred within a year since the formation of suicidal ideation [24]. This result indicates that special attention should be paid to suicidal ideation [25]. Based on the results of the previous and current studies, active intervention should be performed to prevent suicidal behaviors when a target person has both traumatic childhood experiences and suicidal ideation [25-27].

This study has the following limitations. First, the results of this study cannot be generalized to the entire population of college students or young adults because it only focused on college students in a given region (i.e., the Jeju region). Second, this study verified traumatic childhood experiences as a factor that had independent, significant effects on the suicidality of college students. However, given that this study was cross-sectional and based on a certain timeframe, a causal relationship between traumatic childhood experiences and suicidality of college students cannot be accurately identified. Sociodemographic variables were also widely controlled. Third, underreporting or underestimation of symptoms might have measured because self-administered questionnaires were used. More accurate results could have been derived through more objective evaluation and data analysis, using methods such as clinical interviews, expert evaluation, and third-party reporting. However, it should be considered that interviews with those who committed suicide cannot be conducted and that few cases of psychological autopsy have been reported. Therefore, this study is significant since measures for preventing suicidal behaviors can be prepared by analyzing suicide risk factors as performed in this study.

\section{CONCLUSION}

The high suicide rate of young adults is a prominent social problem in Korea, yet little is known about the predictive factors of suicide attempts among them. This study analyzed the relationship between traumatic childhood experiences and suicidality among college students who represent the young adult group. It was found that college students with traumatic childhood experiences showed a high level of suicidality and that such risk increased when they underwent multiple types of traumatic childhood experiences. It was also verified that traumatic childhood experiences of college students had independently significant effects on their suicidal ideation and behaviors. Based on the results of this study, evaluation of traumatic childhood experiences should be implemented among college students especially, those who have suicidal ideation or a history of suicidal attempt and provide them with appropriate mental health services.

\section{CONFLICTS OF INTEREST}

The authors have nothing to disclose.

\section{ACKNOWLEDGEMENTS}

This study was supported by the 2021 education, research and student guidance grant funded by Jeju National University.

\section{ORCID}

\author{
Hyun Ju Yang \\ https://orcid.org/0000-0001-8175-6122 \\ Young Eun Jung \\ https://orcid.org/0000-0001-7608-0009 \\ Joon Hyuk Park \\ https://orcid.org/0000-0002-0396-5284 \\ Moon Doo Kim \\ https://orcid.org/0000-0002-6441-630X
}

\section{SUPPLEMENTARY MATERIAL}

Supplementary material can be found via https://doi. org/10.35986/me.2021.19.3.77.

\section{REFERENCES}

1. Statistics Korea. 2019 Cause of death statistics results. Daejeon: Statistics Korea; 2020.

2. Nock M. Understanding nonsuicidal self-injury: origins, assessment, and treatment. Washington: American Psychological Association; 2009. p. 9-18.

3. Silverman MM, Berman AL, Sanddal ND, O'carroll PW, Joiner TE. Rebuilding the tower of Babel: a revised nomenclature for the study of suicide and suicidal behaviors. Part 
1: background, rationale, and methodology. Suicide Life Threat Behav 2007;37:248-63.

4. Brodsky BS, Stanley B. Adverse childhood experiences and suicidal behavior. Psychiatr Clin North Am 2008;31:22335 .

5. Ministry of Education. School violence survey analysis results announcement. Sejong: Ministry of Education; 2014.

6. Miller AB, Esposito-Smythers C, Weismoore JT, Renshaw KD. The relation between child maltreatment and adolescent suicidal behavior: a systematic review and critical examination of the literature. Clin Child Fam Psychol Rev 2013;16:146-72.

7. Chang E, You SE. Resilience and suicidal behavior in adults with traumatic life events: is resilience a protective factor for suicidal behavior? Korean J Clin Psychol 2014;33:299314.

8. Lee SL. The effects of traumatic event type on posttraumatic growth and wisdom: the mediating effects of social support and coping. Korean J Cult Soc Issues 2013;19:31941.

9. Courtois CA, Ford JD. Treating complex traumatic stress disorders: an evidence-based guide. New York: Guilford Press; 2009.

10. Hermann JL. Trauma and recovery: the aftermath of violence- from domestic abuse to political terror. New York: BasicBooks; 1997.

11. Dube SR, Anda RF, Felitti VJ, Chapman DP, Williamson DF, Giles WH. Childhood abuse, household dysfunction, and the risk of attempted suicide throughout the life span: findings from the Adverse Childhood Experiences Study. JAMA 2001;286:3089-96.

12. Spertus IL, Yehuda R, Wong CM, Halligan S, Seremetis SV. Childhood emotional abuse and neglect as predictors of psychological and physical symptoms in women presenting to a primary care practice. Child Abuse Negl 2003;27:124758.

13. Stein DJ, Chiu WT, Hwang I, Kessler RC, Sampson N, Alonso J, et al. Cross-national analysis of the associations between traumatic events and suicidal behavior: findings from the WHO World Mental Health Surveys. PLoS One 2010;5:e10574.

14. Spitzer RL, Kroenke K, Williams JB. Validation and utility of a self-report version of PRIME-MD: the PHQ primary care study. Primary Care Evaluation of Mental Disorders. Patient Health Questionnaire. JAMA 1999;282:1737-44.

15. Han C, Jo SA, Kwak JH, Pae CU, Steffens D, Jo I, et al. Validation of the Patient Health Questionnaire-9 Korean version in the elderly population: the Ansan Geriatric study. Compr Psychiatry 2008;49:218-23.
16. Afifi TO, Enns MW, Cox BJ, Asmundson GJ, Stein MB, Sareen J. Population attributable fractions of psychiatric disorders and suicide ideation and attempts associated with adverse childhood experiences. Am J Public Health 2008;98:946-52.

17. Choi NG, DiNitto DM, Marti CN, Segal SP. Adverse childhood experiences and suicide attempts among those with mental and substance use disorders. Child Abuse Negl 2017;69:252-62.

18. Cluver L, Orkin M, Boyes ME, Sherr L. Child and adolescent suicide attempts, suicidal behavior, and adverse childhood experiences in South Africa: a prospective study. J Adolesc Health 2015;57:52-9.

19. Perez NM, Jennings WG, Piquero AR, Baglivio MT. Adverse childhood experiences and suicide attempts: the mediating influence of personality development and problem behaviors. J Youth Adolesc 2016;45:1527-45.

20. Nock MK, Borges G, Bromet EJ, Alonso J, Angermeyer M, Beautrais A, et al. Cross-national prevalence and risk factors for suicidal ideation, plans and attempts. Br J Psychiatry 2008;192:98-105.

21. Lee JY, Chun IJ. The pathways from school bullying to depression and suicidal ideation: with a special focus on gender differences. Sch Soc Work J 2013;24:219-39.

22. Kim JY, Jang YE, Lee SJ. A study on the effect of negligence, emotional abuse and physical abuse experience from parents on adolescents' suicidal behavior: mediating effect of committing school violence. Sch Soc Work J 2013;25: 157-83.

23. Felitti VJ, Anda RF, Nordenberg D, Williamson DF, Spitz AM, Edwards V, et al. REPRINT OF: relationship of childhood abuse and household dysfunction to many of the leading causes of death in adults: the adverse childhood experiences (ACE) study. Am J Prev Med 2019;56:774-86.

24. Kessler RC, Borges G, Walters EE. Prevalence of and risk factors for lifetime suicide attempts in the National Comorbidity Survey. Arch Gen Psychiatry 1999;56:617-26.

25. Ports KA, Merrick MT, Stone DM, Wilkins NJ, Reed J, Ebin J, et al. Adverse childhood experiences and suicide risk: toward comprehensive prevention. Am J Prev Med 2017;53:400-3.

26. Stone D, Holland K, Bartholow B, Crosby A, Davis S, Wilkins N. Preventing suicide: a technical package of policy, programs, and practices. Atlanta: Centers for Disease Control and Prevention; 2017.

27. Fortson B, Klevens J, Merrick MT, Gilbert LK, Alexander SP. Preventing child abuse and neglect: a technical package for policy, norm, and programmatic activities. Atlanta: Centers for Disease Control and Prevention; 2016. 